ORIGINAL ARTICLE

\title{
Declining incidence of chickenpox in the absence of universal childhood immunisation
}

\author{
G L Lowe, R L Salmon, D Rh Thomas, M R Evans
}

Arch Dis Child 2004;89:966-969. doi: 10.1136/adc.2002.021618

See end of article for authors' affiliations

.....................

Correspondence to: $G$ L Lowe, National Public Health Service

Communicable Disease

Surveillance Centre, Abton

House, Cardiff CF14 3QX,

UK; Gwen.lowe@nphs.

wales.nhs.uk

Accepted 16 March 2004
Objective: To examine the epidemiology of chickenpox in Wales from 1986 to 2001.

Design: Descriptive analysis of chickenpox consultations reported by the Welsh general practice sentinel surveillance scheme for infectious diseases, compared with annual shingles consultation rates from the same scheme to exclude reporting fatigue and data from a general practice morbidity database to validate results.

Setting: A total of 226884 patients registered with one of 30 volunteer general practices participating in the sentinel surveillance scheme.

Main outcome measures: Age standardised and age specific incidence of chickenpox.

Results: Crude and age standardised consultation rates for chickenpox declined from 1986 to 2001, with loss of epidemic cycling. Rates remained stable in $0-4$ year olds but declined in all older age groups, particularly those aged 5-14 years. Shingles consultation rates remained constant over the same period. Data from the morbidity database displayed similar trends.

Conclusion: General practitioner consultation rates for chickenpox are declining in Wales except in preschool children. These findings are unlikely to be a reporting artefact but may be explained either by an overall decline in transmission or increased social mixing in those under 5 years old, through formal child care and earlier school entry, and associated increasing rates of mild or subclinical infection in this age group. Further investigation, particularly by serological surveillance, is necessary before universal varicella immunisation can be considered in the UK.

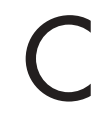

hickenpox results from primary infection with varicella zoster virus. The resulting illness is usually mild, but serious complications and deaths do occur, with the case fatality rate being higher in adults. ${ }^{1}$ A live vaccine against varicella exists, but this is only available for named patients with specific clinical indications in the United Kingdom (UK).

In the United States, clinical reporting of chickenpox has steadily declined since 1987 . However, this decline has been attributed to changes in reporting practices rather than declining incidence ${ }^{2}$ and, as a consequence, universal varicella immunisation at $12-18$ months was introduced in 1995.

Until the early 1990s, data on chickenpox incidence in the UK suggested that age at infection was shifting upwards. ${ }^{3}$ This would potentially increase morbidity and mortality from chickenpox and lend support to the introduction of universal varicella immunisation in the UK also. However, more recent data indicate a marked reversal of this trend, with increasing rates in 0-4 year olds and a decline in older age groups. ${ }^{45}$

To explore these trends further, we analysed age specific consultation rates for varicella in Wales from 1986 to 2001, using data from the Welsh general practice surveillance of infectious diseases scheme, and validated our results against data from a general practice morbidity database (GPMD). We also analysed consultation rates for shingles to exclude the possibility of reporting fatigue by general practitioners (GPs).

\section{METHODS}

The General Practice Surveillance of Infectious Diseases Scheme in Wales is administered by the National Public Health Service Communicable Disease Surveillance Centre (CDSC). ${ }^{6}$ A total of $30 \%$ volunteer practices are included, from all areas of Wales, covering 226884 individuals, or approximately $8 \%$ of the population. The age profile of the combined practice populations matches that of the Welsh population. Practices are contacted weekly and report the number of patients consulting for specific infectious diseases, including chickenpox and shingles. Chickenpox is defined as "a typical disseminated vesicular rash" and shingles as "a typical rash restricted to one or two dermatomes and usually localised pain". Each GP has a notepad for collecting case details that specifies these definitions on the cover. Data on the sex and age group of cases are aggregated at practice level before reporting to CDSC. GPMD data were obtained for comparison. This scheme collects computerised consultation data from 38 volunteer practices in Wales representing approximately $10 \%$ of the population. Four practices are in both schemes.

Annual crude and age specific rates were calculated, the latter based on the total practice population in each age group for each year. Age standardised rates were calculated using the European Standard Population. Cases of unknown age came predominantly from one practice and were allotted to age group in the same proportion as cases of known age. Shingles data were analysed similarly. Trends in consultation rates over time were examined by calculating Kendall's rank correlation coefficient.

\section{RESULTS}

The annual number of chickenpox consultations ranged from 770 to 2605 of which $48.5 \%$ were female, $49.8 \%$ were male, and $1.7 \%$ were of unknown sex. Crude and age standardised consultation rates have declined in Wales since 1986, with loss of epidemic cycling in later years (fig 1). This trend was statistically significant $(p=0.003)$. The mean annual age standardised consultation rate for shingles was $1.76 / 1000$

Abbreviations: CDSC, Communicable Disease Surveillance Centre; GP, general practitioner; GPMD, general practice morbidity database 


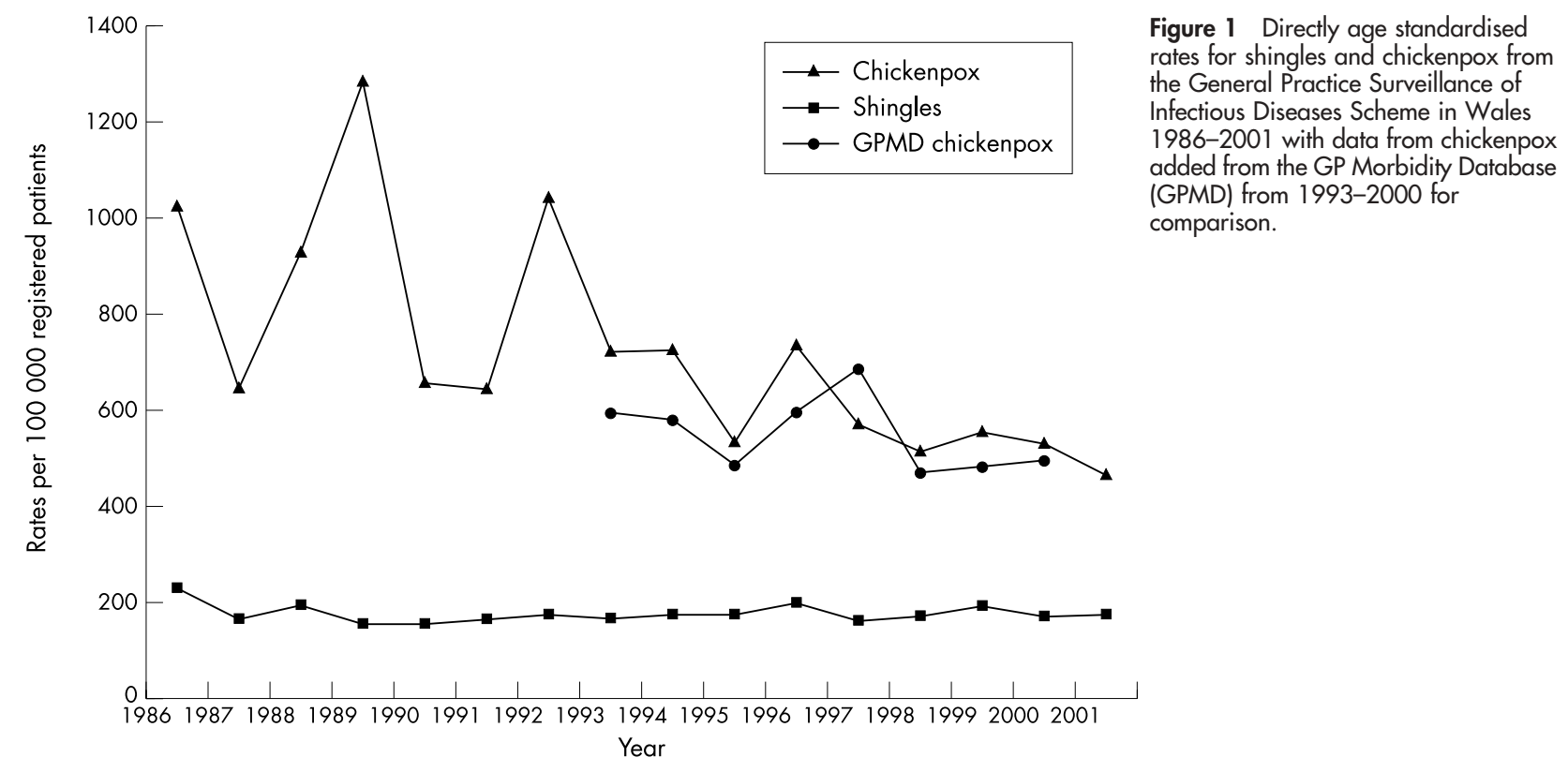

registered patients/year (median 1.73, range 1.59-2.22). The annual shingles consultation rate remained stable throughout the time period $(\mathrm{p}=0.39)$ (fig $\mathrm{l}$ ).

No significant decline occurred in chickenpox incidence rates in children aged $0-4$ years $(p=0.56)$, although there were marked year to year fluctuations, the amplitude of which decreased over time. Rates in the 5-14 year age group fell significantly $(p<0.0001)$ after 1986 and rates in older age groups also fell ( $p=0.003$ for over 35 years), but less steeply (fig 2).

GPMD data for chickenpox and shingles were available for the years 1993-2000. Annual age standardised chickenpox consultation rates were similar to rates reported by the sentinel surveillance scheme (fig 1). Age specific rates showed a similar but less marked trend. The mean age standardised rate for shingles was 2.97/1000 registered patients/year (median 3.00, range 2.43-3.40). Annual shingles rates were approximately one third higher than in the sentinel scheme, but again remained remarkably stable over the time period.

\section{DISCUSSION}

Chickenpox consultation rates from two general practice networks in Wales declined between 1986 and 2001. Rates remained unchanged in 0-4 year olds but declined in all other age groups. By contrast, shingles consultation rates remained constant over the same period. The stable incidence of shingles, a disease caused by reactivation, rather than infection de novo with the varicella zoster virus, suggests the decline in chickenpox is unlikely to be due to reporting

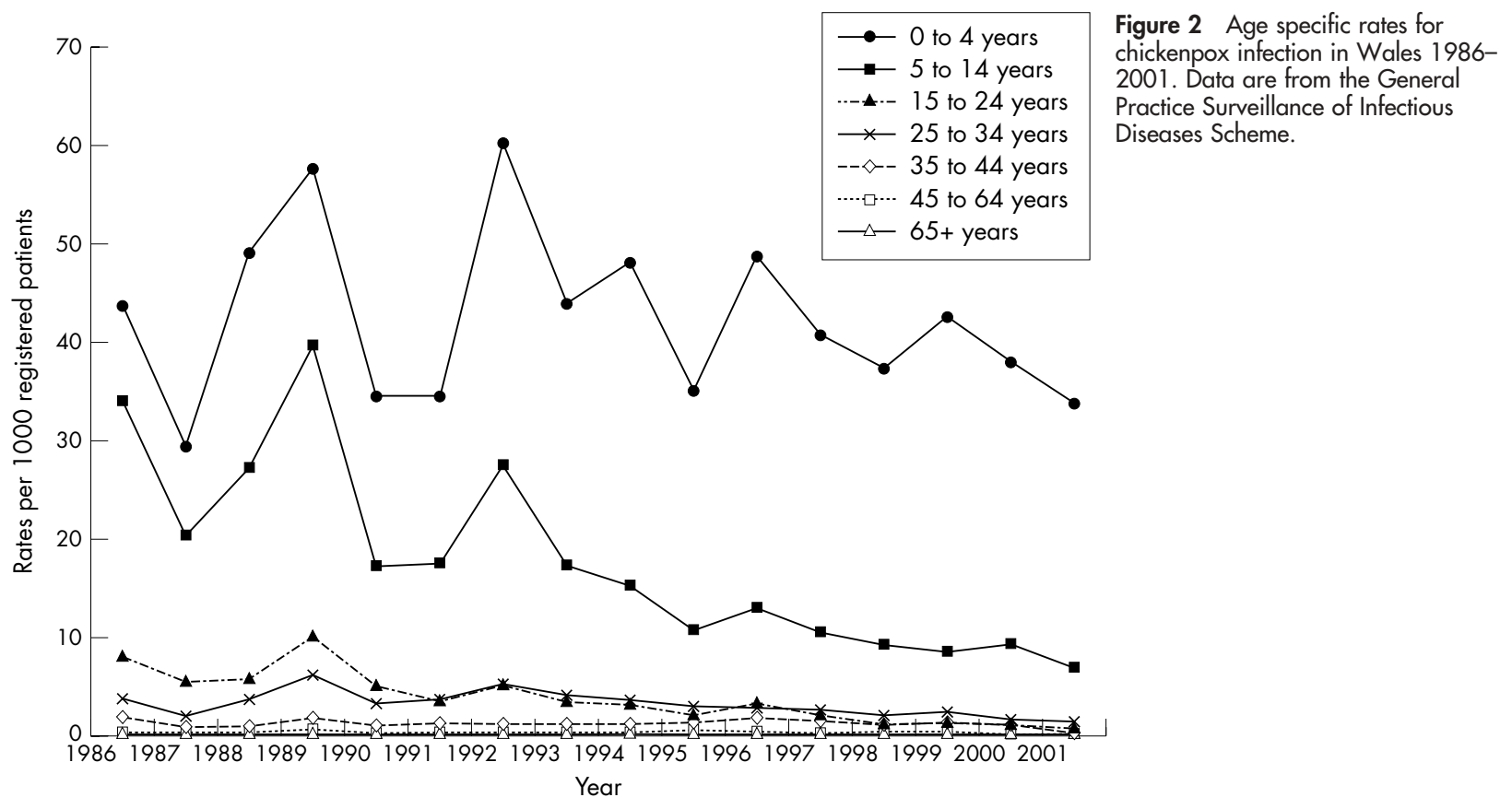


fatigue, as practices are unlikely to tire of reporting one disease selectively to the surveillance system.

Similar trends observed in two general practice networks corroborates the findings. However, both data sources only capture cases brought to the attention of the GP, and do not measure population incidence. Mild and secondary household cases may be missed.

Our results show similarities to surveillance data from Scotland and England. Chickenpox became notifiable in Scotland in 1988 and notifications to 1998 show a rising incidence in $0-4$ year olds, and a decline in older age groups, particularly 5-14 year olds. ${ }^{5}$ English general practice data show similar trends, with a rise in chickenpox consultations in 0-4 year olds between 1980 and $1995^{4}$ followed by a levelling off between 1995 and 2000. ${ }^{7}$ The incidence in 1544 year olds declined in the 1990s after rising throughout the 1980s. ${ }^{4}$

Although in our study, rates in 0-4 year olds have not risen over time, the shift of disease burden into the $0-4$ year age group in proportion to other age groups in the population is a consistent finding. The reason is unknown. That this is found in studies using different data sources and different methods suggests a real phenomenon. The most plausible reason is increased social mixing in pre-schoolers, allowing greater opportunity for virus transmission. ${ }^{8}$ Over the study time period of 1986-2001, births in Wales were stable between 37000 and 39000 from 1986 to 1992, then declined yearly to a low of 31304 in 2000. However, the percentage of children aged under 5 years in school has increased steadily over the last 10 years from nearly $70 \%$ in full or part time school in $1990 / 1991$ to just over $80 \%$ by $2000 / 2001 .{ }^{9}$ Other data show that the number of places in children's day care facilities in Wales increased steadily from 19401 in 1979/80 to 33763 in 1994/95. ${ }^{10}$ This means that pre-school mixing has increased as the birth rate has decreased.

Another possible explanation for the shift of disease burden may be changes in consultation behaviour or severity of chickenpox in different age groups. However, GPMD data showed that although all cause consultation rates in the 04 year age group rose steadily from 1993 to 2000, all cause consultation rates for the 5-14 year age group also rose over the same time period. This suggests that consultation behaviour for chickenpox would have to differ from general consultation trends for this to be the explanation. This is a possibility since chickenpox cases may be actively discouraged from attending GP surgeries because of their infectivity and relatively benign course. If disease severity has reduced markedly, more mild or subclinical cases may be occurring so consultation is less likely. Smaller family sizes may have decreased the severity of chickenpox as secondary family cases are often more severe.

Declining varicella incidence in older age groups could be attributable to a cohort effect, reflecting successive cohorts more likely than their predecessors to be exposed to wider social networks before starting school. ${ }^{8}$ This may be due to increases in the number of pre-school children attending day care over the last few decades and would result in fewer susceptible adults today. However, the alternative possibility that the proportion of varicella zoster virus seronegative adults is increasing cannot be ruled out without seroprevalence studies.

Although a recent study concluded that 25 deaths from chickenpox may occur annually in Britain, ${ }^{1}$ this estimate was based on data from 3 years with significantly high death rates. Analysis over a longer period suggests mortality has fallen, particularly in pre-schoolers. ${ }^{7}$ This supports the observation that chickenpox incidence is changing.

The declining in chickenpox incidence observed in the US before the introduction of universal immunisation was based on reporting to the National Notifiable Disease Surveillance System and has been attributed to reporting artefact. The decrease in states reporting case numbers $\geqslant 3 \%$ of their annual birth cohort was advanced as supporting evidence for this. ${ }^{2}$ We report age specific rates and still demonstrate a decline.

More recent data from the US suggest that varicella declined dramatically in surveillance areas with moderate vaccine coverage. ${ }^{11}$ However, the absence of data from similar areas with no vaccination, and the unexplained national decline recorded before vaccine was introduced mean that this conclusion is by no means certain. It is possible that some or all of the decline in chickenpox incidence could be accounted for by factors other than immunisation.

Should varicella immunisation be introduced in Britain? Experimental studies suggest the vaccine has an efficacy of $85-88.5 \%$ in preventing infection, ${ }^{12}{ }^{13}$ and is $97 \%$ effective in preventing severe disease. ${ }^{12}$ Serious adverse event rates following immunisation are reported to be lower than those after natural infection. ${ }^{14}$ However, the long term effects of vaccination are still under study. Varicella is unusual in that the virus may reactivate to cause herpes zoster. Although the incidence of herpes zoster shortly after vaccination is low $(2.6 / 100000$ doses distributed $),{ }^{14}$ the lifetime cumulative incidence of reactivation is not known. Immunity has persisted and sometimes increased after vaccination, but it is debated whether this represents boosting from exposure to circulating wild virus ${ }^{15} 16$ or reactivation of the latent vaccine virus in the ganglia. ${ }^{17}$ If vaccination reduces but does not eliminate wild virus circulation, and duration of immunity is not life long, a vaccinated cohort may be susceptible as adults when the consequences of infection are more severe.

Large scale serological monitoring of susceptibility in adults would aid in deciding whether to introduce universal immunisation. If most children acquire immunity before school entry, school children and young adults should have higher rates of immunity than their historic counterparts at the same age. Conversely, if chickenpox incidence is declining due to less disease transmission or circulating virus, then adults will become increasingly susceptible and the case for universal varicella immunisation is much stronger.

If our results indicate increasing population immunity, then an alternative strategy would be to selectively test and immunise those at high risk of serious disease, such as pregnant women, health care workers, ${ }^{18}$ and possibly adolescents without a history of chickenpox. The cost effectiveness of immunisation has already been explored for pregnant women. ${ }^{19} 20$ Modelling the impact of different immunisation scenarios using current epidemiological data is necessary.

Our findings highlight the value of actively monitoring varicella incidence. We suggest that adding varicella immunisation to the UK childhood immunisation programme at this stage would be premature. However, in order to corroborate our epidemiological findings, up to date serological surveys are required to establish whether immunity to varicella in older age groups is increasing because chickenpox is now occurring at an earlier age or if the disease incidence is truly waning, so that more older people remain susceptible to the disease.

\section{ACKNOWLEDGEMENTS}

We would like to thank all the general practices that provide weekly returns to the surveillance of infectious diseases scheme in Wales. We also thank Rhian Davey and Richard Lewis of CDSC Wales for collecting and collating this data, and Joe Hunt and Llinos Saxon, from Health Solutions Wales, Cardiff, for providing us with GPMD data. 


\section{CONTRIBUTORS}

GL analysed the data and wrote the first draft of the paper. DT undertook statistical testing. RS, DT, and ME contributed to interpreting the data and revising the paper. GL will act as guarantor.

\section{Authors' affiliations}

G L Lowe, R L Salmon, D Rh Thomas, M R Evans, National Public Health Service Communicable Disease Surveillance Centre, Abton House, Cardiff CF14 3QX, UK

Conflict of interest: none declared.

\section{REFERENCES}

1 Rawson H, Crampin A, Noah N. Deaths from chickenpox in England and Wales 1995-7: analysis of routine mortality data. BM/ 2001 323:1091-3.

2 CDC. Evaluation of varicella reporting to the National Notifiable Disease Surveillance System - United States, 1972-1997. MMWR Morb Mortal Wkly Rep, 1999;48:55-8.

3 Fairley CK, Miller E. Varicella-zoster virus epidemiology - a changing scene? $J$ Infect Dis 1996;174(suppl 3):S314-9.

4 Ross AM, Fleming DM. Chickenpox increasingly affects pre-school children. Commun Dis Public Health 2000;3:213-5.

5 Bramley JC, Jones IG. Epidemiology of chickenpox in Scotland: 1981-1998. Commun Dis Public Health 2000;3:282-7.

6 Palmer SR, Smith RMM. GP surveillance of infections in Wales. Commun Dis Rep 1991;1:R25-8.

7 Brisson M, Edmunds WJ, Gay NJ, et al. Deaths from chickenpox in adults are decreasing. BMJ 2002;324:609.
8 Holmes SJ, Morrow AL, Pickering LK. Child-care practices: effects of social change on the epidemiology of infectious diseases and antibiotic resistance. Epidemiol Rev 1996;18:10-28.

9 National Assembly for Wales. A statistical focus on children in Wales 2002. Cardiff: National Assembly for Wales, 2002.

10 National Assembly for Wales. Schools in Wales: general statistics 2001. Cardiff: National Assembly for Wales, 2001.

11 Seward JF, Watson BM, Peterson CL, et al. Varicella disease after introduction of varicella vaccine in the United States, 1995-2000. JAMA 2002;287:606-11.

12 Vazquez M, LaRussa PS, Gershon AA, et al. The effectiveness of the varicella vaccine in clinical practice. N Engl J Med 2001;344:955-60.

13 Vessy SJ, Chan CY, Kuter BJ, et al. Childhood vaccination against varicella: persistence of antibody, duration of protection, and vaccine efficacy. J Pediatr 2001;139:297-304

14 Centers for Disease Control and Prevention. Prevention of varicella: updated recommendations of the Advisory Committee on Immunisation Practices (ACIP). MMWR Morb Mortal Wkly Rep, 1999;48(RR-6):3-4.

15 LaRussa $P$, Steinberg SP, Shapiro E, et al. Varicella vaccine revisited. Nat Med 2000;6:1299.

16 Seward J, Jumaan A, Schmid S. Varicella vaccine revisited. Nat Med 2000;6:1298-9.

17 Krause PR, Klinman DM. Varicella vaccination: evidence for frequent reactivation of the vaccine strain in healthy children. Nat Med 2000;6:451-4.

18 Devi R, Muir D, Rice P. Healthcare workers should not be forgotten. BMJ 2002;324:610.

19 Smith WJ, Jackson LA, Watts DH, et al. Prevention of chickenpox in reproductive-age women: cost-effectiveness of routine prenatal screening with postpartum vaccination of susceptibles. Obstet Gynecol 1998;92:535-45.

20 Glantz JC, Mushlin Al. Cost-effectiveness of routine antenatal varicella screening. Obstet Gynecol 1998;91:519-28.

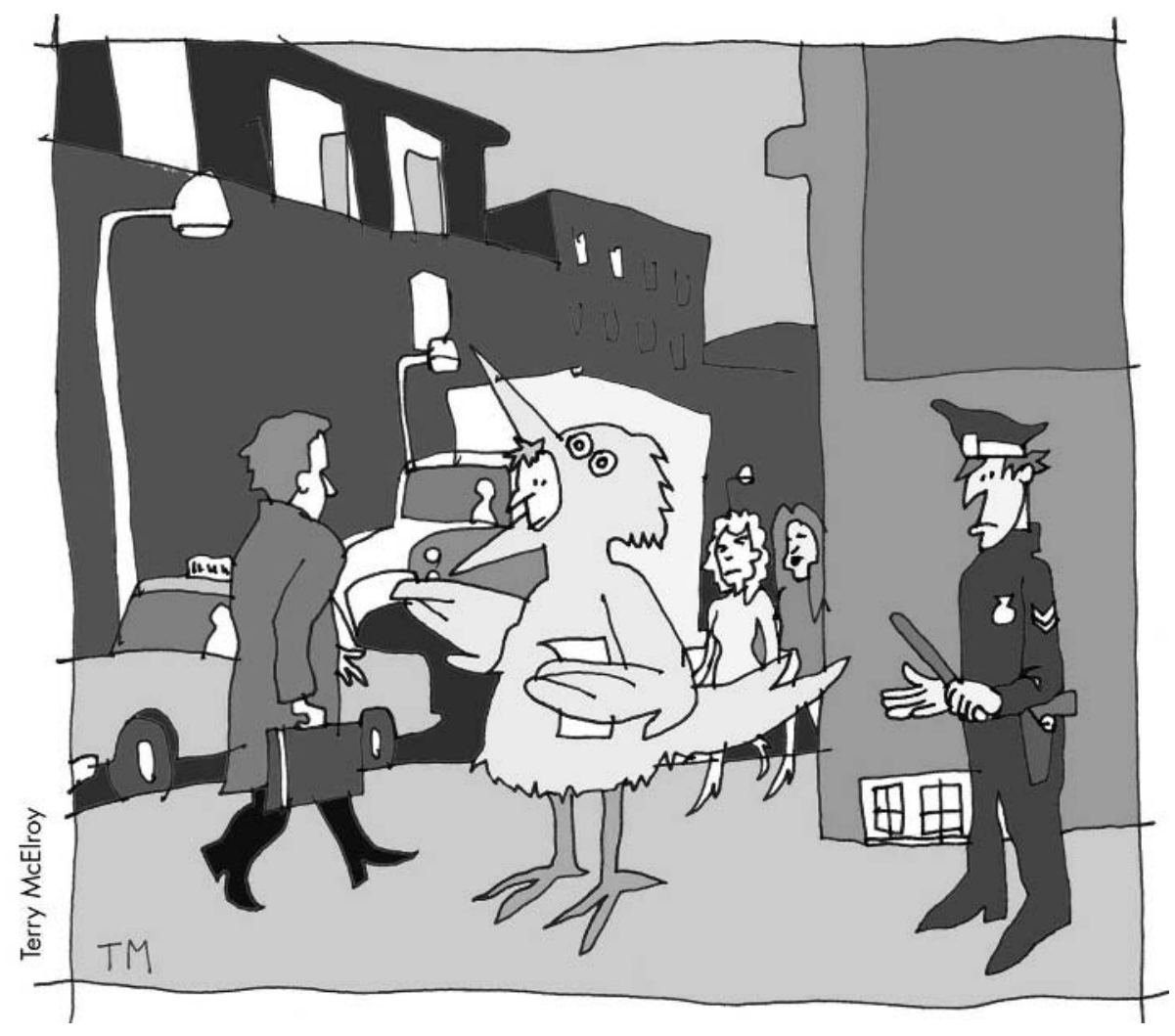

